\title{
Evaluating the Effect of Periodontal Therapy on Hematological Parameters among Subjects with Chronic Periodontitis: An Interventional Study
}

\author{
${ }^{1}$ Sarita Parihar, ${ }^{2}$ Naresh Kumar, ${ }^{3}$ Atul Bhatnagar, ${ }^{4} \mathrm{Ajit}$ V Parihar
}

\begin{abstract}
Aim: With advancements in science and technology, our knowledge and understanding about the pathogenesis of periodontal disease and its impact on systemic health of humans has increased. The aim of the present study is to evaluate the effect of periodontal therapy on hematological parameters among subjects with chronic periodontitis.
\end{abstract}

Materials and methods: A total of 42 systemically healthy male patients with chronic periodontitis with age group between 30 and 55 years were included in the study. Hematological parameters evaluated from peripheral blood samples at baseline were hemoglobin $(\mathrm{Hb})$ level, erythrocyte count [red blood cells (RBC)], erythrocyte sedimentation rate (ESR), mean corpuscular volume (MCV), mean corpuscular hemoglobin $(\mathrm{MCH})$, and mean corpuscular hemoglobin concentration $(\mathrm{MCHC})$. Periodontal parameters like plaque index $(\mathrm{PI})$, gingival index (GI), probing pocket depth (PPD), and clinical attachment loss $(\mathrm{CAL})$ were recorded at baseline. Periodontal therapy including surgery, if required, was carried out in all the patients. Periodontal clinical parameters were repeated at 6 and 12 months. The hematological parameters again were recorded at the end of 12 months.

Results: Data analyses showed statistically significant improvements in $\mathrm{Hb}$ levels and erythrocyte counts after periodontal therapy. Decreased value of ESR after treatment indicated resolution of periodontal inflammation. There was nonsignificant improvement in MCV value, and much lesser improvement in $\mathrm{MCH}$ and $\mathrm{MCHC}$ values.

\footnotetext{
${ }^{1,4}$ Assistant Professor, ${ }^{2,3}$ Professor

${ }^{1}$ Department of Periodontics, Faculty of Dental Sciences Institute of Medical Sciences, Banaras Hindu University Varanasi, Uttar Pradesh, India

${ }^{2}$ Department of Oral and Maxillofacial Surgery, Faculty of Dental Sciences, Institute of Medical Sciences, Banaras Hindu University, Varanasi, Uttar Pradesh, India

${ }^{3}$ Department of Prosthodontics, Faculty of Dental Sciences Institute of Medical Sciences, Banaras Hindu University Varanasi, Uttar Pradesh, India

${ }^{4}$ Department of Orthodontics, Faculty of Dental Sciences Institute of Medical Sciences, Banaras Hindu University Varanasi, Uttar Pradesh, India
}

Corresponding Author: Sarita Parihar, Assistant Professor Department of Periodontics, Faculty of Dental Sciences, Institute of Medical Sciences, Banaras Hindu University, Varanasi, Uttar Pradesh, India, Phone: +918004925677, e-mail: drsarita6@ gmail.com
Conclusion: The result of the present study shows that periodontal therapy leads to an improvement in hematological parameters in chronic generalized periodontitis patients. It can also conclude that chronic periodontitis can lead to anemia, like other chronic diseases.

Keywords: Anemia, Cytokines, Hemoglobin, Periodontitis.

How to cite this article: Parihar S, Kumar N, Bhatnagar A, Parihar AV. Evaluating the Effect of Periodontal Therapy on Hematological Parameters among Subjects with Chronic Periodontitis: An Interventional Study. J Oral Health Comm Dent 2018;12(1):25-30.

Source of support: Nil

Conflict of interest: None

\section{INTRODUCTION}

The advances in science and technology have increased our knowledge and understanding of the pathogenesis toward periodontal diseases. Periodontal diseases are basically infectious diseases, but environmental, physical, social, and host factors may affect and modify the disease expression. Chronic periodontitis, being the most prevalent form of periodontitis, can be clinically diagnosed by the detection of chronic inflammatory changes in the marginal gingiva, presence of periodontal pockets, loss of clinical attachment, and radiographically by observation of bone loss. ${ }^{1}$

Therefore, a two-way relationship exists in which the periodontal disease of an individual may have an effect on an individual's systemic health or the systemic disease may influence an individual's periodontal status.

Considerable scientific data indicate that the localized infections that are characteristic of chronic periodontitis can have a significant effect on the systemic health of humans and animals. ${ }^{2-5}$ With the periodontal tissues initiating an inflammatory response to bacteria and their products, systemic challenges with these agents also evoke a major vascular response or better termed as the host response. Infections, malignant cells, and autoimmune dysregulation all provoke the activation of the immune system and production of cytokines, especially tumor necrosis factor (TNF)- $\alpha$ and interleukin (IL)- 1 and IL-6. ${ }^{6}$ These inflammatory cytokines can depress erythropoietin production leading to the development of anemia.7,8

The anemia of chronic disease (ACD)is defined as the anemia that occurs in chronic infections,inflammatory 
conditions or neoplastic disorders that is not due to marrow deficiencies or other diseases and occurs despite the presence of adequate iron stores and vitamins. ${ }^{9}$

Hutter et $\mathrm{al}^{10}$ suggested that periodontitis needs to be considered as a chronic disease that may cause a decrease in the number of erythrocytes and consequently lower hemoglobin concentrations $(\mathrm{Hb} \%)$ in a substantial number of patients. The most prominent characteristic of anemia of chronic disease (ACD) is the development of disturbances of iron homeostasis with increased uptake and retention of iron within the cells of the reticuloendothelial system.

A small attempt, however, is required to know whether treating periodontal disease locally will have an effect on systemic conditions. Thus, the present study was taken up to evaluate the effect of periodontal therapy on hematological parameters on subjects with chronic generalized periodontitis.

\section{MATERIALS AND METHODS}

The present study was done as a 12-month follow-up (longitudinal cohort study) comprising 42 adult male subjects (aged 30-55 years) with chronic generalized periodontitis, who were voluntarily recruited from the outpatient section of the Department of Periodontics, Faculty of Dental Sciences, Institute of Medical Sciences (IMS), Benares Hindu University. Subjects with chronic periodontitis were screened until a final sample size of 42 was achieved, who fulfilled the inclusion criteria of having pocket probing depth (PPD) $\geq 5 \mathrm{~mm}$ at $30 \%$ of sites and CAL $\geq 2 \mathrm{~mm}$ at $30 \%$ of sites. ${ }^{1}$

Systemic and family history were recorded in detail. Subjects suffering from any (1) acute or chronic medical conditions, except chronic periodontitis, (2) subjects having a history of blood loss, (3) present or past smokers, (3) subjects who have undergone periodontal therapy, (4) subjects who have used antibiotics or oral supplementation in the previous 6 months prior to enrollment, and (5) subjects having less than 16 remaining teeth in the mouth were excluded.

The research protocol was submitted to the Institutional Ethical Committee of IMS. After ethical approval, all subjects were verbally informed about the Study Protocol, and a written informed consent was obtained.

\section{Study Flow Chart}

At the first visit, an informed verbal/written consent was taken from the subjects and signs of inflammation were recorded using PI and GI. The PPD and CAL were recorded using the World Health Organization probe. After recording the periodontal status, blood samples were obtained from all patients for hematological investigations. The patients were treated as per routine periodontal therapy and followed for a period of 12 months. Patients were neither put on any iron or vitamin supplements nor any modifications in the diet introduced. Periodontal parameters were again recorded after 6 months and 1 year; however, hematological investigations were repeated at the end of 1 year only (Flow Chart 1).

All the examinations were carried out by a single skilled dental professional. Intraexaminer reliability was 0.81 for PI, 0.83 for GI, 0.79 for PPD, and 0.82 for CAL.

\section{Red Blood Cells Analyses}

Under aseptic measures, $5 \mathrm{~mL}$ of venous blood sample was drawn by venipuncture from antecubital fossa between 9:00 AM and 12:00 noon using a $5 \mathrm{~mL}$ syringe. The collected sample was divided into two parts, the first one for hematological parameter analysis and the second one for serum ferritin estimation. Samples collected were immediately transported to the laboratory for analysis of $\mathrm{Hb} \%$, number of erythrocytes, $\mathrm{MCV}, \mathrm{MCH}$, and MCHC. The samples were analyzed on a fully automated hematologic analyzer. The ESR was measured according to Wintrobe's method. Serum ferritin estimation was done in Mindray BS-800 analyzer. Only patients with serum ferritin value above $30 \mathrm{ng} / \mathrm{mL}$ were included in the present study in order to exclude patients with pure iron deficiency anemia.

\section{Statistical Analyses}

The collected data were subjected to statistical analysis through Statistical Package for the Social Sciences (statistical presentation system software). The statistical methods applied were paired $t$ test, repeated measures analysis of variance (ANOVA), and descriptive statistics. The paired sample $t$ test procedure was used to compare the means of variables with two readings. Repeated measures ANOVA procedure was used for the parameters having more than two readings. The results were presented in text, tables, and graphs.

\section{RESULTS}

Statistical evaluation of clinical and hematological parameters gave the following results. Table 1 and Graphs 1 to 5 show the mean, standard deviation, repeated measures ANOVA of PI scores, GI score, PPD score, and CAL score at baseline, 6 months, and 1 year and the result was found highly significant statistically.

The mean plaque score was 1.74, 1.00, 0.84 at baseline, 6 months, and 1 year follow-up respectively. When repeated measures ANOVA was applied, there was statistically significant difference in plaque scores. Least plaque score was seen at 1 year follow-up followed by 6 months $[p<0.000$, highly significant (HS)]. 
Flow Chart 1: Study protocol

Screening of subjects with chronic generalized periodontitis

Investigations

Red blood cell analysis

Hemoglobin concentration

ESR

Mean corpuscular volume

Mean corpuscular hemoglobin

Mean corpuscular hemoglobin concentration

Few were not eligible and few did not give consent

Assessed for eligibility for chronic periodontitis $(\mathrm{N}=42)$

Scaling and root planing done and surgical therapy performed wherever required

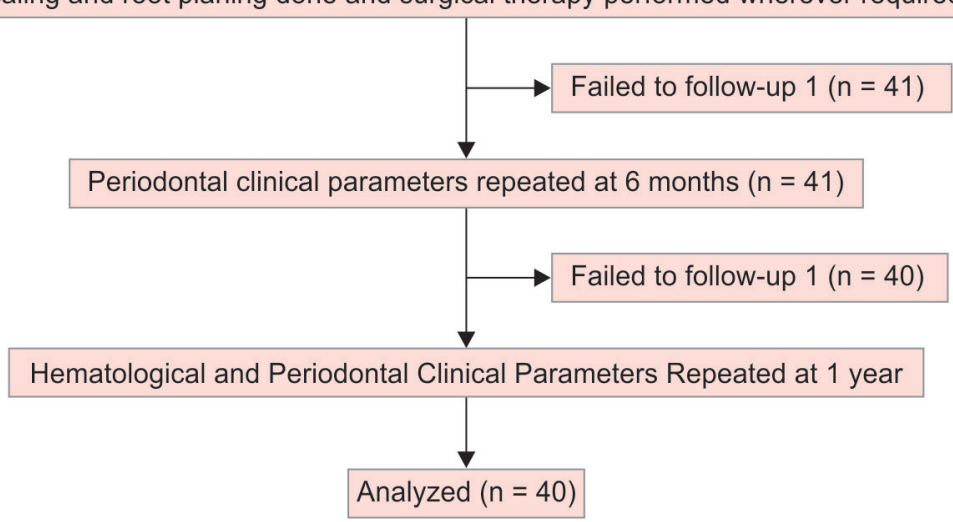

Table 1: Mean, SDs, and repeated measures ANOVA of PI, GI, PPD, and CAL

\begin{tabular}{llllll}
\hline Visits & $n$ & $P l($ mean $\pm S D)$ & Gingival index (mean $\pm S D)$ & $P P D($ mean $\pm S D)$ & $C A L(m e a n \pm S D)$ \\
\hline Baseline (a) & 42 & $1.74 \pm 0.3$ & $1,75 \pm 0.19$ & $5.20 \pm 0.40$ & $2.5 \pm 0.55$ \\
6 months (b) & 41 & $1.01 \pm 0.29$ & $1.08 \pm 0.23$ & $3.77 \pm 0.47$ & $2.15 \pm 0.48$ \\
1 year (c) & 40 & $0.84 \pm 0.14$ & $0.82 \pm 0.14$ & $2.70 \pm 0.51$ & $1.20 \pm 0.46$ \\
Repeated measures ANOVA & & $\mathrm{F}=134.9$ & $\mathrm{~F}=249$ & $\mathrm{~F}=285$ & $\mathrm{~F}=57.72$ \\
& & $\mathrm{p}=0.000$ & $\mathrm{p}=0.000$ & $\mathrm{p}=0.000$ & $\mathrm{p}=0.000$ \\
& & $\mathrm{c}<\mathrm{b}<\mathrm{a}$ & $\mathrm{c}<\mathrm{b}<\mathrm{a}$ & $\mathrm{c}<\mathrm{b}<\mathrm{a}$ & $\mathrm{c}<\mathrm{b}<\mathrm{a}$ \\
\hline
\end{tabular}

SD: Standard deviation

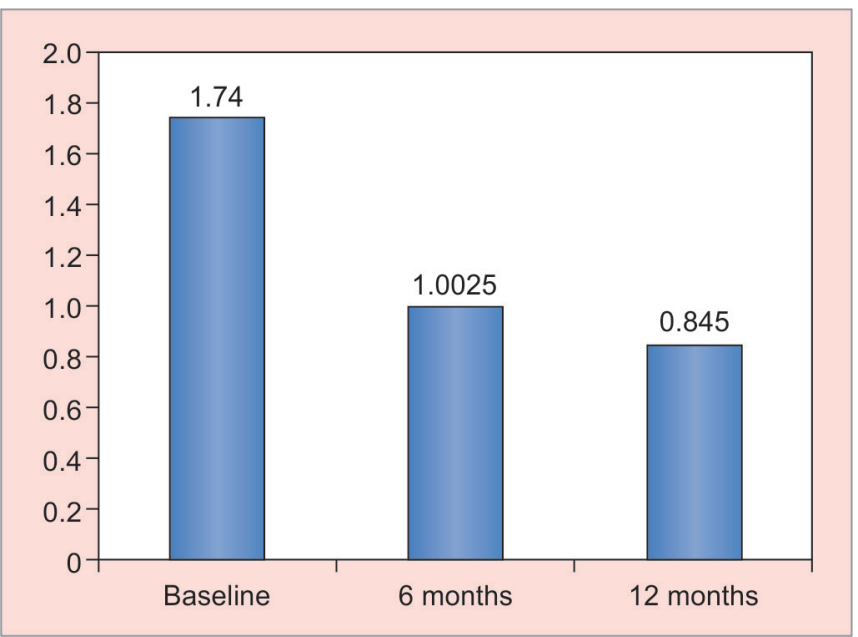

Graph 1: Comparison of mean PI scores

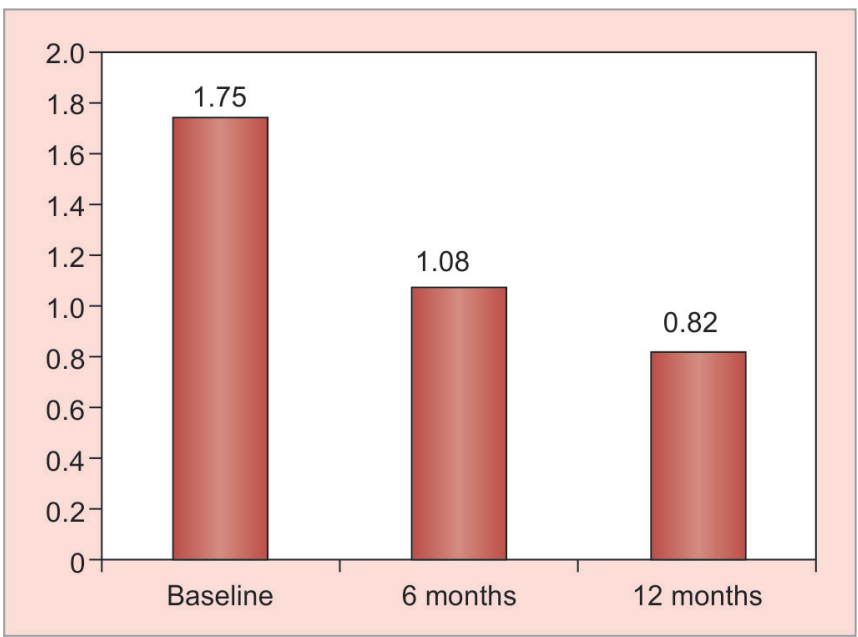

Graph 2: Comparison of mean GI scores 


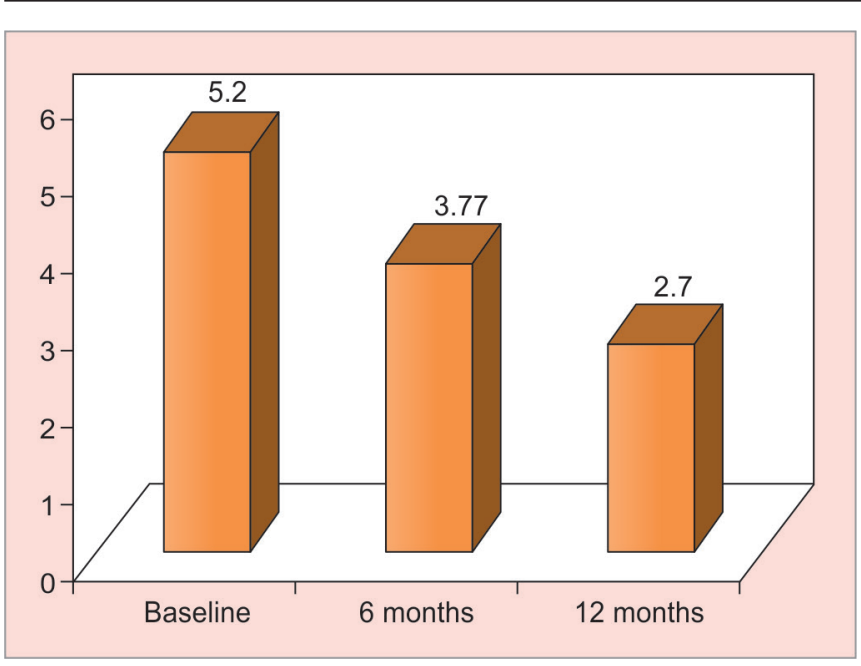

Graph 3: Comparison of mean PPDs

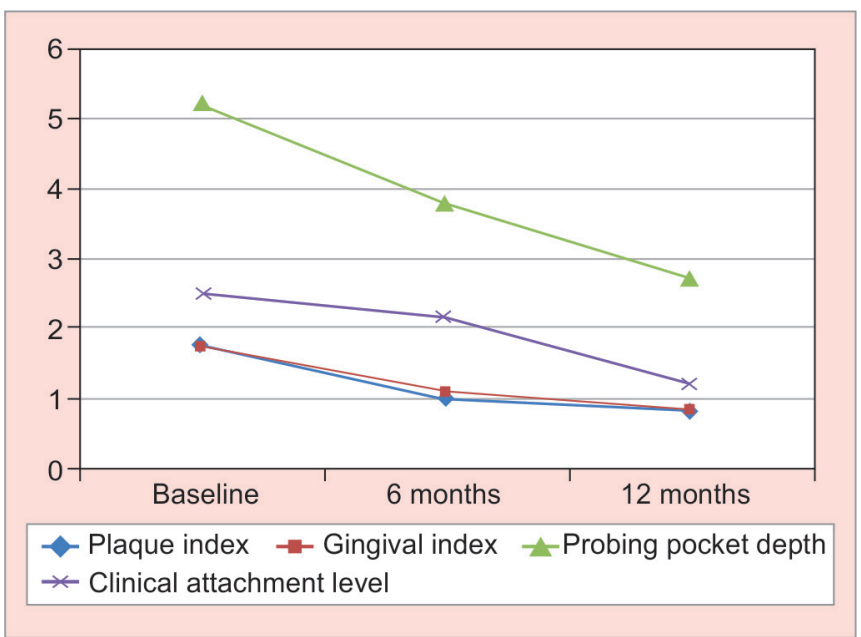

Graph 5: Comparison of means of periodontal clinical parameters

The mean gingival score was $1.75,1.08,0.82$ at baseline, 6 months, and 1 year follow-up respectively. When repeated measures ANOVA was applied, there was statistically significant difference in GI scores. Least gingival score was seen at 1 year follow-up followed by 6 months ( $p<0.000$, HS).

The mean PPD score was 5.2, 3.77, 2.7 at baseline, 6 months, and 1 year follow-up respectively. When repeated measures ANOVA was applied, there was statistically significant difference in plaque scores. Least plaque score was seen at 1 year follow-up followed by 6 months $(\mathrm{p}<0.000, \mathrm{HS})$.

The mean CAL score was 2.5, 2.15, and 1.20 at baseline, 6 months, and 1 year follow-up respectively. When repeated measures ANOVA was applied, there was

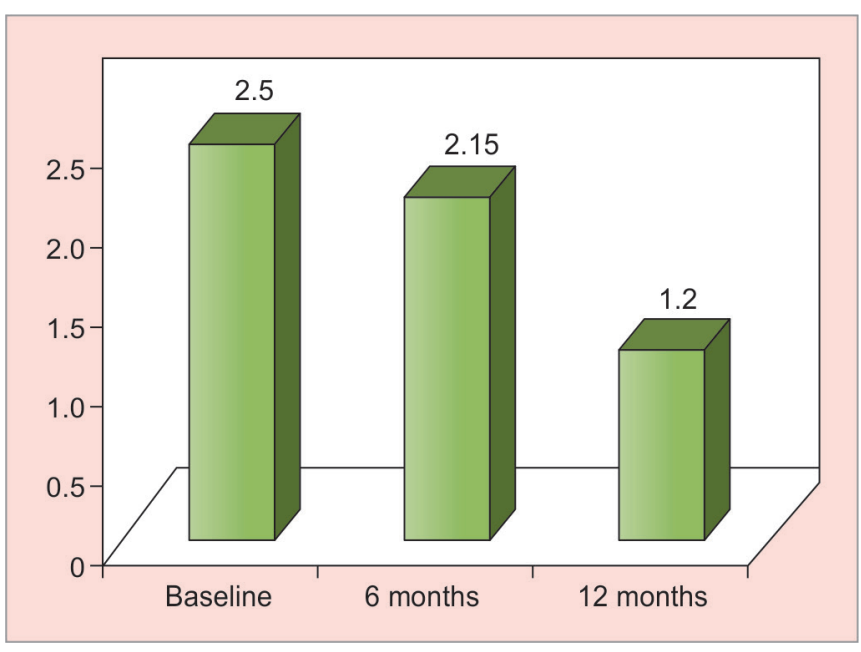

Graph 4: Comparison of mean CAL scores

statistically significant difference in CAL scores. Least CAL score was seen at 1 year follow-up followed by 6 months ( $\mathrm{p}<0.000, \mathrm{HS})$.

The $\mathrm{Hb}, \mathrm{RBC}, \mathrm{ESR}, \mathrm{MCV}, \mathrm{MCH}$, and $\mathrm{MCHC}$ were $13.47,4.63,22.02,86.86,29.0$ and 32.58 at baseline and $14.71,5.02,11.32,87.33,30.27$ and 33.36 at the end of 1 year respectively. The $t$ test was used to analyze the difference between 1 year and baseline values of various hematological parameters. All parameters of blood analyses showed improvement over 1 year. The difference was statistically significant for all the parameters except $\mathrm{MCV}$, which showed no statistical differences from baseline to 1 year (Table 2, Graphs 6 and 7).

\section{DISCUSSION}

The purpose of the present interventional study was to evaluate the hematologic status of patients with chronic periodontitis and assess the effect of periodontal therapy on hematological parameters. Only patients with serum ferritin above $30 \mathrm{ng} / \mathrm{mL}$ were included in the study. This was done to exclude patients with pure iron deficiency anemia. ${ }^{10}$ Smokers were excluded because smoking is considered a cofactor for the development of periodontitis ${ }^{11-13}$ and anemia. ${ }^{14}$ Women were excluded from the study due to failure to adjust to menopausal status and blood loss due to menstrual cycle.

Previous studies have reported a tendency toward anemia in patients with chronic periodontitis, ${ }^{15-19}$ whereas a reverse relationship was presented in the data collected

Table 2: Paired samples $t$ test of hematological parameters

\begin{tabular}{lllllll}
\hline Visits & $H b \%(\mathrm{gm} \%)$ & $R B C\left(\right.$ million $\left./ \mathrm{mm}^{3}\right)$ & $E S R(\mathrm{~mm})$ & $M C V(f L)$ & $M C H(\mathrm{pg})$ & $M C H C(\mathrm{~g} / \mathrm{dL})$ \\
\hline Baseline & $13.47 \pm 1.0$ & $4.63 \pm 0.40$ & $22.02 \pm 5.4$ & $86.86 \pm 5.8$ & $29.0 \pm 2.6$ & $32.58 \pm 0.90$ \\
1 year & $14.71 \pm 0.7$ & $5.02 \pm 0.37$ & $11.32 \pm 1.9$ & $87.33 \pm 3.4$ & $30.27 \pm 1.9$ & $33.36 \pm 0.89$ \\
t-value & 13.65 & 10.30 & 16.15 & 0.60 & 6.54 & 8.04 \\
p-value & $<0.001$ & $<0.001$ & $<0.001$ & 0.54 & $<0.001$ & $<0.001$ \\
\hline
\end{tabular}




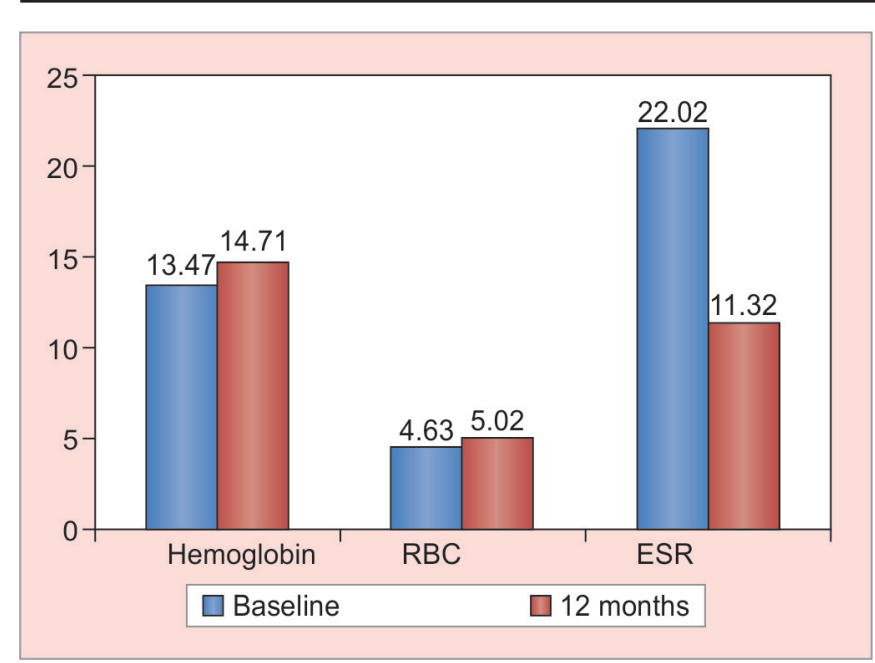

Graph 6: Comparison of means of $\mathrm{Hb}, \mathrm{RBC}$, and ESR

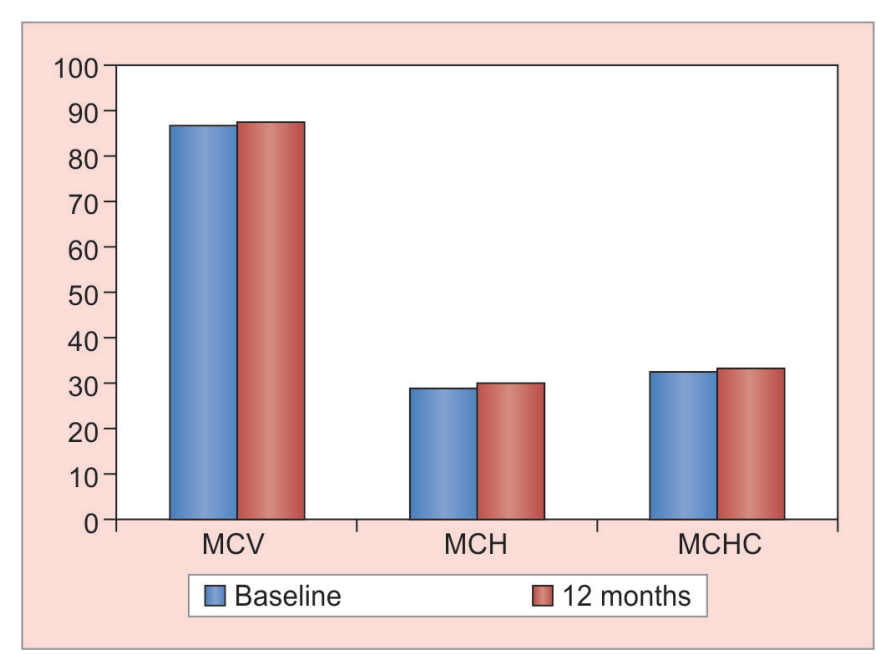

Graph 7: Comparison of means of $\mathrm{MCV}, \mathrm{MCH}$, and $\mathrm{MCHC}$

during the third national health and nutrition examination survey (NHANES III), which proposed that individuals with anemia may be more likely to have periodontal disease. ${ }^{20}$ Few studies failed to show any association between $\mathrm{Hb}$ level and periodontal status. ${ }^{21,22}$ Chronic periodontitis subjects with no history of systemic disease and reduced $\mathrm{Hb}$ and erythrocyte counts suggest that chronic periodontitis should be considered as a possible cause or contributing factor for ACD.

For any inflammatory condition, ESR is considered an important parameter. In our study, increased values of ESR at baseline suggests that chronic periodontitis has an inflammatory component in it and decrease in ESR values after periodontal treatment shows reduction of periodontal inflammation. ${ }^{10}$

In chronic periodontitis subjects, reduced level of $\mathrm{Hb}$ and erythrocyte is thought to be caused by upregulation of the proinflammmatory cytokine. Proinflammatory cytokines, such as TNF- $\alpha$, IL- $1 \beta$, interferon $\gamma$, and prostaglandin E2 are found in high concentrations in inflamed periodontal tissues. The various cytokines can enter the blood circulation and affect distant sites and organs. ${ }^{1}$ The same inflammatory cytokines have been found to be central in the pathogenesis of ACD. ${ }^{10}$ It has been suggested that hepcidin is a primary factor in the pathogenesis of ACD, which is a cytokine-mediated anemia commonly faced in clinical practice and characterized by hypoferremia with adequate reticuloendothelial iron stores. ${ }^{23}$ A previous study ${ }^{24}$ indicated that IL-6 mediates hepcidin increases, resulting in hypoferrremia during inflammation. Kemna et $\mathrm{al}^{25}$ showed the importance of the IL-6-hepcidin axis in the development of hypoferremia in inflammation and focused on the rapid responsiveness of this iron regulatory system.

Our study exhibits a change in mean $\mathrm{Hb}$ value by 1.24 $\mathrm{mg} / \mathrm{dL}(\mathrm{p}<0.001)$ and a mean increase of 0.37 million/

$\mathrm{mm}^{3}$ in erythrocyte count at the end of 12 months after periodontal therapy. The results are in accordance with a previously reported interventional study. ${ }^{26}$ Other observational studies also reported reduced $\mathrm{Hb} \%, \mathrm{RBC}$, and other hematocrit values in chronic periodontitis patients as compared with healthy controls. ${ }^{10,27}$ Minimal increase in values of $\mathrm{MCV}, \mathrm{MCH}$, and $\mathrm{MCHC}$ after periodontal intervention suggest normocytic and normochromic anemia not due to iron or vitamin deficiency. ${ }^{28}$ The present study shows statistically highly significant change in $\mathrm{Hb}$ and $R B C$ values, but not as high as seen in anemia due to other systemic inflammatory conditions like rheumatoid arthritis ${ }^{29}$ and multiple myeloma, ${ }^{30}$ hence concluding that anemia due to chronic periodontitis is mild.

The present study shows improvement in all hematologic parameters in subjects with chronic generalized periodontitis from baseline to 12 months. These can be interpreted on the supposition that as time progresses, further reductions in periodontal inflammation lead to improvement in hematologic parameters ${ }^{19}$; hence, the resolution of periodontal inflammation with improvement in hematologic parameters provides evidence that periodontal therapy can improve the anemic status of subjects with chronic periodontitis.

\section{CONCLUSION}

Chronic systemic conditions have a direct effect on the general health and well-being of an individual. The present study suggests that chronic periodontitis may cause reduction of $\mathrm{Hb}$ concentration and $\mathrm{RBC}$ count and provides evidence that periodontal therapy improved the hematologic status of subjects with chronic periodontitis. The present study also strengthens the evidence that periodontitis does have various systemic effects. However, considering the limited sample size and lack of evaluation of the relationship between severity of 
periodontitis and anemia in the present study, future studies remain to be conducted, which will further reduce the bias occurring due to other confounding factors as well as provide a better and more thorough view of the real situation.

\section{REFERENCES}

1. Newman MG, Takei HH, Klokkevold PR, Carranza FA. Clinical periodontology. 10th ed. St. Louis (MO): W.B. Saunders; 2006.

2. DeStefano F, Anda RF, Kahn HS, Williamson DF, Russell CM. Dental disease and risk of coronary heart disease and mortality. BMJ 1993 Mar;306(6879):688-691.

3. Kweider M, Lowe GD, Murray GD, Kinane DF, McGowan DA. Dental disease, fibrinogen and white cell count; links with myocardial infarction? Scott Med J 1993 Jun;38(3):73-74.

4. Syrjänen J, Peltola J, Valtonen V, Iivanainen M, Kaste M, Huttunen JK. Dental infections in association with cerebral infarction in young and middle-aged men. J Intern Med 1989 Mar;225(3):179-184.

5. Collins JG, Smith MA, Arnold RR, Offenbacher S. Effects of Escherichia coli and Porphyromonas gingivitis lipopolysaccharide on pregnancy outcome in the golden hamster. Infect Immun 1994 Oct;62(10):4652-4655.

6. Weiss G, Goodnough LT. Anemia of chronic disease. N Engl J Med 2005 Mar;352(10):1011-1023.

7. Faquin WC, Schneider TJ, Goldberg MA. Effect of inflammatory cytokines on hypoxia-induced erythropoietin production. Blood 1992 Apr;79(8):1987-1994.

8. Raja KB, O Latunde-Dada G, Peters TJ, McKie AT, Simpson RJ. Role of interleukin-6 in hypoxic regulation of intestinal iron absorption. Br J Haematol 2005 Dec;131(5):656-662.

9. Lee GR. The anemia of chronic disease. Semin Hematol 1983 Apr;20(2):61-80.

10. Hutter JW, van der Velden U, Varoufaki A, Huffels RA, Hoek FJ, Loos BG. Lower numbers of erythrocytes and Lower levels of hemoglobin in periodontitis patients compared to control subjects. J Clin Periodontol 2001 Oct;28(10):930-936.

11. Genco RJ, Löe H. The role of systemic conditions and disorders in periodontal disease. Periodontal 20001993 Jun;2:98-116.

12. Genco RJ. Current view of risk factors for periodontal diseases. J Periodontal 1996 Oct;67(Suppl 10):1041-1049.

13. Bergström J, Preber H. Tobacco use as a risk factor. J Periodontol 1994 May; 65(Suppl 5):545-550.

14. Erdemir EO, Nalcaci R, Caglayan O. Evaluation of systemic markers related to anemia of chronic disease in the peripheral blood of smokers and non-smokers with chronic periodontitis. Eur J Dent 2008 Apr;2:102-109.
15. Siegel EH. Total erythrocyte, leukocyte and differential white cell counts of blood in chronic periodontal disease. J Dent Res 1945 Oct;24:270-271.

16. Lainson PA, Brady PP, Fraleigh CM. Anemia, a systemic cause of periodontal disease? J Periodontol 1968 Jan;39(1):35-38.

17. Chawla TN, Kapoor KK, Teotia SP, Singh NK. Anemia and periodontal disease: a correlative study. J Indian Dent Assoc 1971 Apr;43(4):67-78.

18. Gokhale SR, Sumanth S, Padhye AM. Evaluation of blood parameters in patients with chronic periodontitis for signs of anemia. J Periodontol 2010 Aug;81(8):1202-1206.

19. Pradeep AR, Anuj A. Anemia of chronic disease and chronic periodontitis: does periodontal therapy have an effect on anemic status. J Periodontol 2011 Mar;82(3):388-394.

20. Kazarian MC, Janus CE, Abbott DM, Waldrop TC, Rogers JD. Associations between anemia and periodontitis: a NHANES III review. IJDR Abstract no. 2968.

21. Wakai K, Kawamura T, Umemura O,Hara Y, Machida J,Anno T, Ichihara Y, Mizuno Y, Tamakoshi A, Lin Y, et al. Associations of medical status and physical fitness with periodontal disease. J Clin Periodontol 1999 Oct;26(10):664-672.

22. Havemose-Poulsen A, Westergaard J, Stoltze K, Skjødt H, Danneskioldsamsøe B, Locht H, Bendtzen K, Holmstrup P. Periodontal and hematological characteristics associated with aggressive periodontitis, juvenile idiopathic arthritis, and rheumatoid arthritis. J Periodontal 2006 Feb;77(2):280-288.

23. Means RT. Hepcidin and cytokines in anemia. Hematology 2004 Oct-Dec;9(5-6):357-362.

24. NemethE, RiveraS, Gabayan V,KellerC, TaudorfS, Pedersen BK, Ganz T. IL-6 mediates hypoferremia of inflammation by inducing the synthesis of the iron regulatory hormone hepcidin. J Clin Invest 2004 May;113(9):1271-1276.

25. KemnaEH,PickkersP,NemethE, van derHoevenH,SwinkelsD. Time-course analysis of hepcidin, serum Iron and plasma cytokine levels in humans injected with LPS. Blood 2005 Sep;106(5):1864-1866.

26. Agarwal N, Kumar VS, Gujjari SA. Effect of periodontal therapy on haemoglobin and erythrocyte levels in chronic generalized periodontitis patients: an interventional study. J Indian Soc Periodontal 2009 Jan;13(1):6-11.

27. Kolte RA, Kolte AP, Deshpande NM. Assessment and comparison of anemia of chronic disease in healthy subjects and chronic periodontitis patients: a clinical and hematological study. J Indian Soc Periodontol 2014 Mar-Apr;18(2):183-186.

28. Mohan, H. Textbook of pathology. 5 th ed. New Delhi: Jaypee brothers Medical Publishers (P) ltd; 2005.

29. Kelly WN, Harris ED Jr, Ruddy S, Sledge CB. Textbook of rheumatology. 5th ed. Philadelphia (PA): WB Saunders; 1997.

30. Steensma D. Prospects and perils of retrospective risk assessment of erythropoiesis stimulating agents. Am J Hematol 2008 Sep;83(9):693-694. 